\title{
THROMBOSIS AND FACTOR VII ACTIVITY
}

\author{
BY \\ L. POLLER \\ From the Clinical Laboratory, the Royal Infirmary, Liverpool*
}

(RECEIVED FOR PUBLICATION DECEMBER 11, 1956)

The tests for the coagulation time of the blood which are in general use have contributed little to the understanding of abnormal tendencies to thrombosis, as they are extremely insensitive to all but gross changes in the coagulation mechanism. A variety of techniques have been evolved to meet this defect. One group of these methods depends on the addition of an inhibitor, e.g., heparin, to the routine clotting time method (Waugh and Ruddick, 1944 ; Silverman, 1948).

It has been shown, using a heparin sensitized method (Poller, 1956), that statistically the coagulation time is significantly shortened 48 hours after a thrombo-embolic episode. The drawbacks to the use of such methods as simple prediction tests have been discussed (Poller, 1955).

As thromboplastin and heparin are to a large extent mutually antagonistic in action, and shortened heparin plasma clotting times have been found in the first few days after operation when tissue damage and other factors may cause an increase in thromboplastin activity, it may be supposed that increased resistance to heparin may be related to the ability to produce thromboplastin in the plasma of such patients. An investigation of various aspects of thromboplastin production was therefore performed in a group of patients and parallel controls. Prothrombin activity, factor V and VII activity, and the production of thromboplastin in the thromboplastin generation test were studied in both groups, together with heparin plasma clotting times. In view of the recently established effect of chylomicra on the coagulation time (Poole, 1955), serum cholesterol values were determined in both groups as a feature of secondary interest.

\section{Subjects}

These were two groups. The first consisted of 20 cases of recent thrombosis within 48 hours of clinical onset. Eighteen were cases of coronary thrombosis and two of thrombophlebitis. The second or control group was made up of 20 hospital patients

*Present address : General Hospital, Warrington without clinical evidence of thrombosis. Ten were cases of congestive cardiac failure and 10 ambulant surgical patients before operation.

\section{Investigations}

Prothrombin Times.-The Quick one-stage procedure (1935) was used.

Patients with thrombosis were paired with equal numbers of controls. Tests were performed together with the same set of reagents. Results were expressed directly in seconds.

Factor V.-Factor V activity was determined quantitatively using the principle outlined by Biggs and Macfarlane (1953).

Stored plasma which gave a single-stage prothromi bin time of 45 seconds was employed as a diluent for. the test plasma. The same batch of factor-V-deficiefs plasma was used throughout the investigations. Dilur tions from 10 to $50 \%$ of test plasma were prepared. Parallel tests on paired patients and controls were performed with the same reagents.

Factor VII.-Quantitative factor VII determinations $\overrightarrow{0}$ were performed with a technique identical to that for factor $\mathrm{V}$ determinations apart from the substitution of "dindevan" plasma for stored plasma. Walker and Hunter (1954) have shown that "dindevan" acts mainly on factor VII. The factor-VII- $\frac{?}{0}$ deficient plasma gave a prothrombin time of $60 \mathrm{sec}-\frac{\overline{0}}{3}$ onds and the same plasma was used throughout the investigations. The effect of storage was overcome of by pairing patients and controls throughout the series.

Thromboplastin Generation.-The method of Biggs 음 and Macfarlane (1953) was used.

Venous blood was collected from a case of recent $\frac{7}{2}$ thrombosis and a second specimen from a control from which citrated plasma and serum were obtained $\mathcal{N}$ following the usual technique. Fresh normal serum $N$ was obtained from the paired control and stored serum was not used in this study.

The same solution of alumina was used throughout. This was found to prolong the single-stage prothrom-o bin time to about 75 seconds after three minutes at $\frac{2}{\Phi}$ $37^{\circ} \mathrm{C}$. Normal platelet suspensions were used and $\stackrel{0}{?}$ were prepared in the usual manner. The suspensions 0 were used for two days and then rejected.

Plasma from the control subject or from the pre- $\frac{\vec{\Phi}}{\mathbb{Q}}$ paration of platelets was used as the substrate. 
Thromboplastin dilution curves were prepared on both the thrombotic patient and the control. To facilitate the statistical analysis, readings were expressed as a percentage of the maximum thromboplastin concentration of the patient with thrombosis.

Heparin Plasma Clotting Times.-These were performed using the method previously described (Poller, 1954). The principle is that plasma containing a standard amount of heparin is recalcified at $37^{\circ} \mathrm{C}$. after a fixed time interval from collection. The same solution of heparin was used throughout the investigation.

Serum Cholesterol.-Sackett's (1925) method was used. The specimens for cholesterol estimation were taken as soon as possible after onset and all within 48 hours. The control series specimens were taken two or three hours after a meal.

\section{Results}

The means of the readings in the various tests are given in the table, together with the standard deviation and standard error of difference between

TABLE

RESULTS

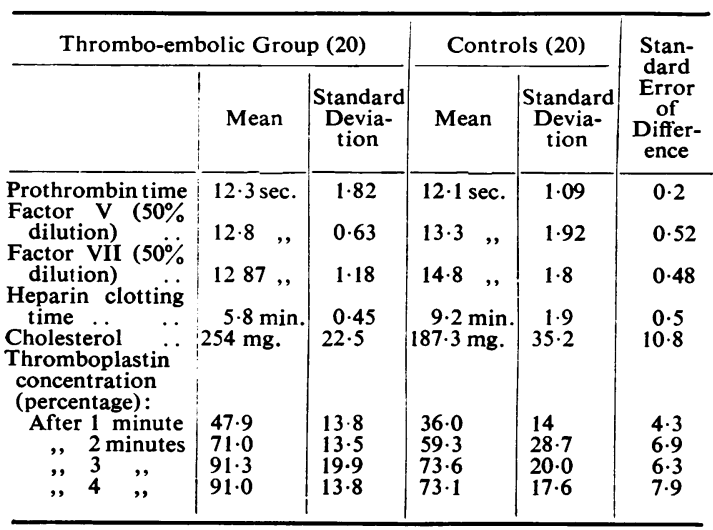

readings in the two groups. The level of significance was taken as three times the standard error of the difference.

Prothrombin Times.-The slight increase in mean prothrombin time for the group with thrombosis is not significant.

Factor $\mathbf{V}$ Levels.-The effect of dilution of test plasma with factor-V-deficient plasma may be seen in Fig. 1. Although there appears to be greater factor $\mathrm{V}$ activity in the patients with thrombosis at all dilutions, the differences are small, however, and not statistically significant.

Factor VII.-The effect of dilution with factorVII-deficient plasma is seen in Fig. 2. Here the differences are more pronounced and statistically significant at all dilutions. The figures for the $50 \%$ dilutions are given in the accompanying table. An important feature of this increased factor VII activity was that it was present in all 20 cases of thrombosis. All the sera of the thrombotic group showed marked activity in the thromboplastin generation test. The distribution of the readings for the $50 \%$ dilution may be observed in the histogram (Fig. 3).

Thromboplastin Generation.-The rates and relative concentrations of thromboplastin over the first four minutes are given. There is a suggestion that the concentration of thromboplastin may be greater in the group with thrombosis. It only approaches significance after one minute, however, and the increase was only in 11 of the 20

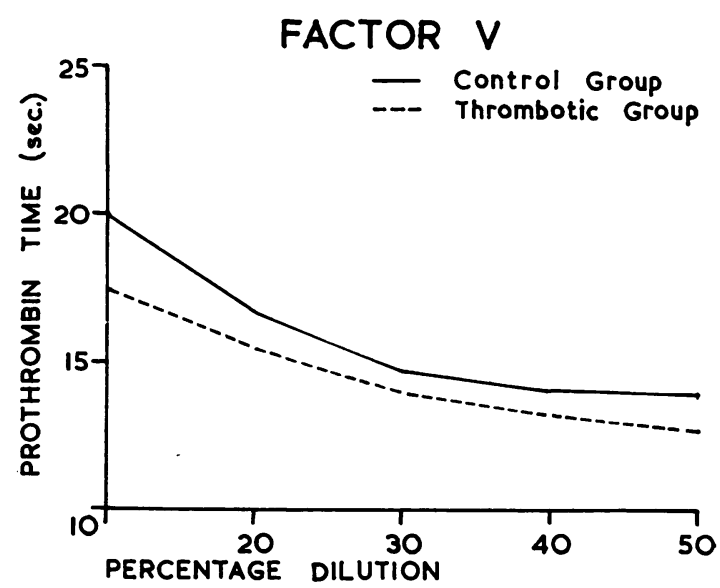

FIG. 1.-Mean readings for dilution by factor-V-deficient plasma in thrombotic and control groups.

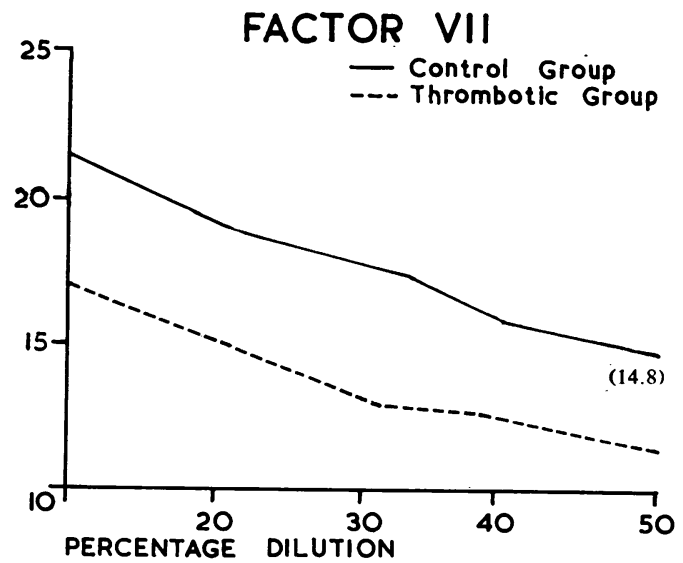

FIG. 2.-Mean readings for dilution by factor-VII-deficient plasma in thrombotic and control groups. 


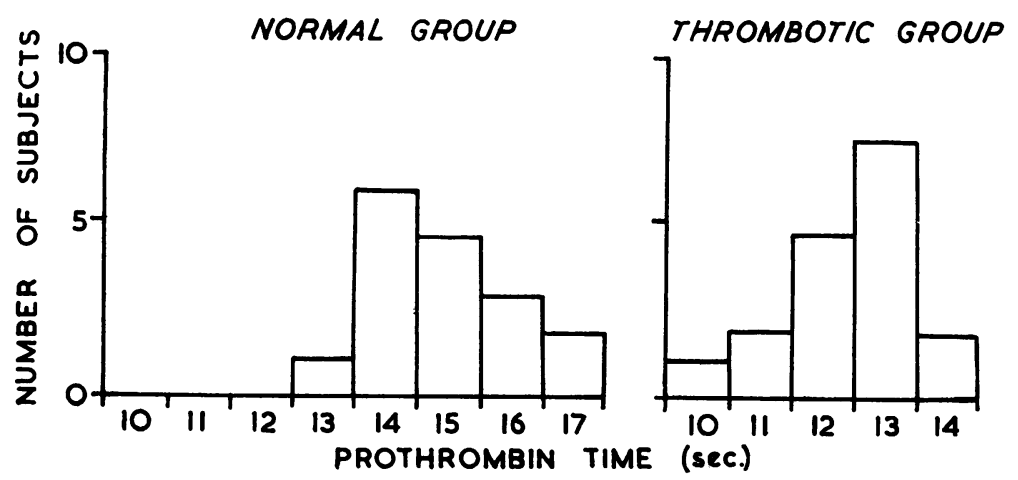

FIG. 3.-The effect of dilution with factor-VII-deficient plasma on one-stage prothrcmbin time in normal and thrombotic groups. cases of thrombo-embolism. This lack of consistency may perhaps be due to the fact that the test is unsuited for this type of experiment, as in some cases the effect was very marked when compared with the control.

Heparin Plasma Clotting Times.-The figures for the two groups are seen in the table.

The shortening of the clotting time in thrombosis was very significant and analogous to previous results on larger series (Poller, 1956).

Cholesterol.-The values for cholesterol estimations are also given in the table. There are some cases in the group with thrombosis with very high values over $350 \mathrm{mg}$. As a result there was a significant difference between the groups of patients and controls although only 10 of 20 patients had levels over $220 \mathrm{mg}$. Thus increased cholesterol, like increased thromboplastin generation, could not be regarded as a constant finding in these patients.

\section{Discussion}

The most striking finding which has not previously been recorded is the high level of factor VII activity in cases of recent thrombosis when contrasted with paired controls. The clinical importance of raised factor VII activity in relation to thrombo-embolism is suggested by the work of Koller, Loeliger, and Duckert (1952), Ciulla and Santoni (1954), and Alexander, Meyers, Goldstein, Gurewich, and Grinspoon (1954), who recorded increased activity during pregnancy. This was believed to be in accord with the concept of hypercoagulability in pregnancy. Furthermore, Wessler (1955) has produced rapid thrombosis in dogs by the infusion of a serum preparation rich in S.P.C.A. (factor VII) activity.
The role of factor VII as an essential activator of thromboplastin formation was generally accepted until quite recently and was supported by results in several cases of congenital deficiency of this factor. Recent case reports by Hicks (1955), Goldstein and Alexander (1955), and Jürgens (1955) suggest that thromboplastin generation is normal in such cases.

It is possible, therefore, that the shortened heparin clotting time in recent thrombosis is not related to the role of factor VII in thromboplastin production but depends on a specific anti-heparin activity of factor VII which is described in another paper being prepared by the author.

The presence of raised factor VII activity provides a firm basis for the administration of drugs of the coumarin group, which reduce the level of activity of this factor in addition to that of prothrombin.

\section{Summary}

High levels of factor VII activity when compared with paired control subjects were noted in a group of cases suffering from thrombotic disorders within 48 hours of onset, together with accelerated heparin clotting times. Increased thromboplastin generation and raised serum cholesterols were less constant findings. The clinical importance of increased factor VII activity is discussed.

Gratitude is expressed to Dr. Rosemary Biggs for help in the preparation of this paper.

\section{REFERENCES}

Alexander, B., Meyers, L., Goldstein, R., Gurewich, V., and Grinspoon, L. (1954). J. clin. Invest., 33, 914.

Biggs, R., and Douglas, A. S. (1953). J. clin. Path., 6, 23. and Macfarlane, R. G. (1953). Human Blood Coagulation. Blackwell, Oxford.

Ciulla, U., and Santoni, G. (1954). Haematologica, 38, 575

Goldstein, R., and Alexander, B. (1955). Fed. Proc., 14, 219.

Hicks, N. D. (1955). Med. J. Aust., 2, 331.

Hicks, N. D. (1955). Acta haemat. (Basel), 14, 57.

Koller, F., Loeliger, H., and Duckert, F.(1952). Rev. Hémat., 7, 156 Koller, F., Loeliger, H., and Duckert,

Poller, L. (1954). Angiology, 5, 21. 14, 372. (1956). Clin. Sci., 15, 55.

Poole, J. C. P. (1955). Brit. J. Haemat., 1, 229.

Quick, A. J. (1935). J. biol. Chem., 109, Ixxiii.

Sackett, G. E. (1925). Ibid., 64, 203.

Silverman, S. B. (1948). Blood, 3, 147.

Walker, W., and Hunter, R. B. (1954). J. clin. Path., 7, 249.

Waugh, T. R., and Ruddick, D. W. (1944). Canad. med. Ass. J. augh, T. R.

Wessler, S. (1955). J. clin. Invest., 34, 647. 